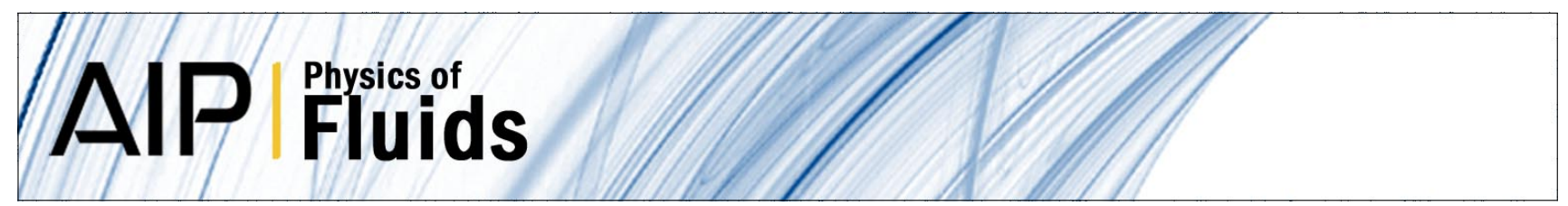

\title{
Discretevelocity collision dynamics for polyatomic molecules
}

David B. Goldstein

Citation: Phys. Fluids A 4, 1831 (1992); doi: 10.1063/1.858404

View online: http://dx.doi.org/10.1063/1.858404

View Table of Contents: http://pof.aip.org/resource/1/PFADEB/v4/i8

Published by the American Institute of Physics.

\section{Related Articles}

High accuracy numerical solutions of the Boltzmann Bhatnagar-Gross-Krook equation for steady and oscillatory Couette flows

Phys. Fluids 24, 032004 (2012)

Rare backflow and extreme wall-normal velocity fluctuations in near-wall turbulence

Phys. Fluids 24, 035110 (2012)

Binary scattering model for Lennard-Jones potential: Transport coefficients and collision integrals for nonequilibrium gas flow simulations

Phys. Fluids 24, 027101 (2012)

Admissible shock waves and shock-induced phase transitions in a van der Waals fluid

Phys. Fluids 23, 086101 (2011)

Rarefied gas dynamics on a planetary scale

Phys. Fluids 23, 030608 (2011)

\section{Additional information on Phys. Fluids A}

Journal Homepage: http://pof.aip.org/

Journal Information: http://pof.aip.org/about/about_the_journal

Top downloads: http://pof.aip.org/features/most_downloaded

Information for Authors: http://pof.aip.org/authors

\section{ADVERTISEMENT}

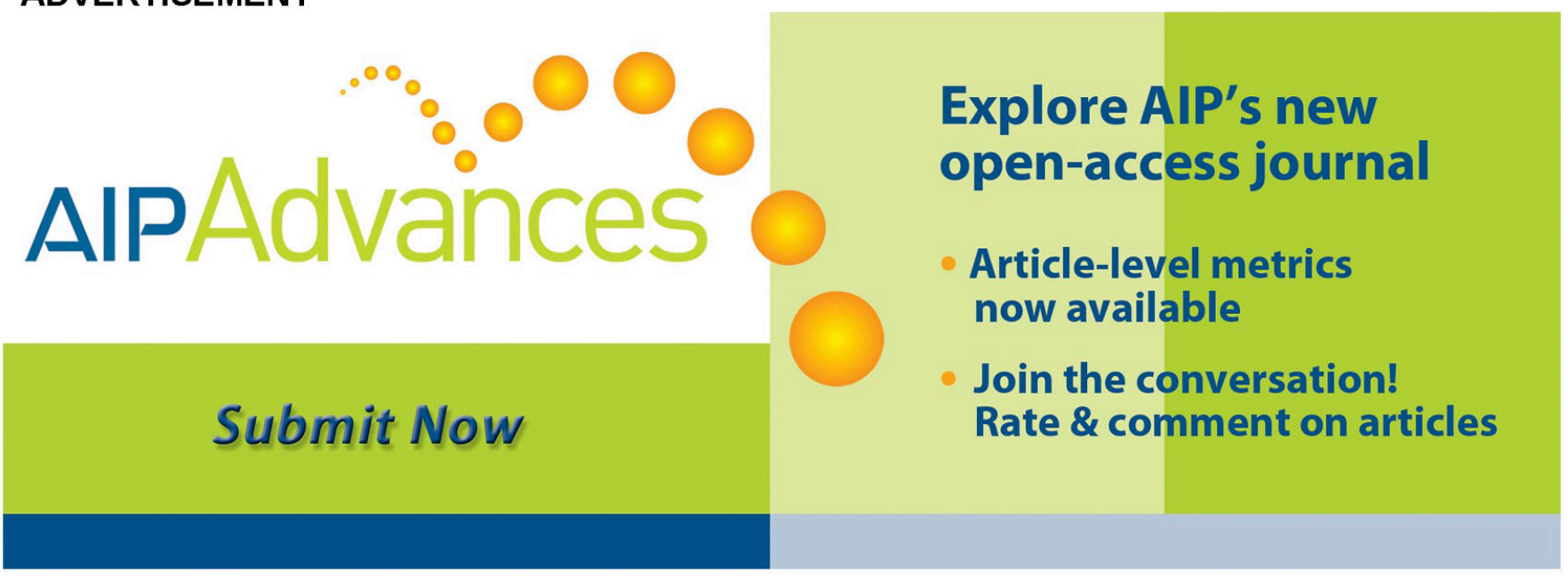




\title{
Discrete-velocity collision dynamics for polyatomic molecules
}

\author{
David B. Goldstein ${ }^{\text {a) }}$ \\ GALCIT, California Institute of Technology, Pasadena, California 91125
}

(Received 26 November 1991; accepted 30 March 1992)

\begin{abstract}
A multispeed discrete-velocity molecular model of a rarefied gas is developed which permits quantitatively accurate simulation of mixtures of molecules of different mass which have both translational and internal rotational energies. In particular, the molecules are represented as having three discrete velocity components and a single discrete internal energy that does not directly affect the translational motion. During binary collisions, rotational and translational kinetic energies are exchanged according to a phenomenological model. Results are presented for the thermal relaxation to equilibrium of mixtures of $\mathrm{H}_{2}$ and $\mathrm{Ne}, \mathrm{N}_{2}$ and $\mathrm{O}_{2}$, and $\mathrm{N}_{2}$ and $\mathrm{CO}_{2}$. Good agreement is found with the corresponding continuousvelocity results.
\end{abstract}

\section{INTRODUCTION}

The Boltzmann equation, an integrodifferential equation, describes the evolution of the number of molecules having a particular velocity in a flowing gas. Evaluation of the collision integral, which describes the gain and loss of particles of a given velocity, is notably difficult. The equations of radiative transport, which are similar to the Boltzmann equation, can be greatly simplified by supposing that light travels along discrete rays or streams, ${ }^{1}$ thus reducing the equations to a set of coupled differential equations. That assumption leads to the discrete ordinate method used to compute radiation transport through matter. ${ }^{2}$ Gross ${ }^{3}$ suggested the possibility of applying a discretization approach to the Boltzmann equation and Broadwell, ${ }^{4-6}$ citing such ideas, developed a model of a molecular gas in which particle velocity components are discrete; the molecules can move only with velocities $\mathbf{c}_{i}$, which belong to a finite set. The evolution of the number of molecules at each point in velocity space can then be described by a differential equation. Broadwell used two different discretizations of velocity space; in one the particles move with unit speed in the six directions parallel to the Cartesian axes and in the other they move in the eight directions along lines at $45^{\circ}$ to the axes (Fig. 1). Particle collisions [where particles of type $\left(\mathbf{c}_{i}, \mathbf{c}_{j}\right)$ become particles of type $\left.\left(\mathbf{c}_{k}, \mathbf{c}_{l}\right)\right]$ were modeled by probabilistic scattering rules that were incorporated into the gain and loss terms of the equation. The collisions, which exactly conserve mass, momentum, and energy, only occur between particles that lie on diametrically opposite sides of a unit sphere centered at the origin of velocity space. Broadwell studied shock wave structure and low Mach number Couette and Rayleigh flows; in both cases, unexpectedly good correspondence between the extremely simple model and more refined theory was found.

For this model, Caflisch and Papanicolaou ${ }^{7}$ have since derived Euler and Navier-Stokes equations and Caflisch ${ }^{8}$ has investigated shock wave profiles. A general discussion

\footnotetext{
a) Present Address: Center for Fluid Mechanics, Turbulence, and Computation, Brown University, Box 1966, Providence, Rhode Island 02912.
}

of discrete-velocity gases, including the Broadwell model, is given in the monograph by Gatignol ${ }^{9}$ and the review paper by Platkowski and Illner, ${ }^{10}$ with particular emphasis on the mathematical subtleties. Recent studies of the discrete-velocity Boltzmann equation have investigated the relationship between the resulting continuum fluid transport coefficients and their corresponding continuousvelocity values, ${ }^{11}$ and have greatly expanded the volume of velocity space that can be considered. ${ }^{12,13}$

If space and time are discretized, a discrete-velocity model may be simulated as a cellular automaton (CA). In such a simulation, particles travel along links between lattice sites in space and collide with other particles encountered at those sites. The simplicity of the set of collision rules that specify collision outcomes which may conveniently be simulated generally restricts the CA models to low velocity resolution, that is, only a few velocities. Also, for technical reasons, an exclusion principle requiring that two particles may not occupy the same point in phase space is applied to each lattice site. CA simulations model an inherently compressible gas, but when it is desired to avoid the pathologies resulting from compressibility they have generally been applied in the low Mach number, in compressible limit.

CA methods have been applied to several problems, including shear flow between parallel plates, ${ }^{14}$ shock waves, ${ }^{15}$ the Rayleigh problem and flow over a flat plate normal to the flow, ${ }^{16}$ flow about a cylinder and KelvinHelmholtz instability, ${ }^{17}$ and for reactive systems. ${ }^{18}$ The advantages of the CA are that simulations can be performed quickly using Boolean algebra and may even be directly computed in specialized hardware. Additionally, because flow boundaries can be easily specified, complex geometries can be simulated. ${ }^{19}$ The drawbacks are that the methods may not be more efficient than conventional finitedifference techniques for continuum flows and may not yield results consistent with the Navier-Stokes equations; low resolution of phase space can lead to spurious conservation laws ${ }^{20}$ and to anisotropy of the stress tensor. ${ }^{21} \mathrm{Also}$, to simulate a gas pressure that is independent of fiow velocity it is necessary to consider particles having many 


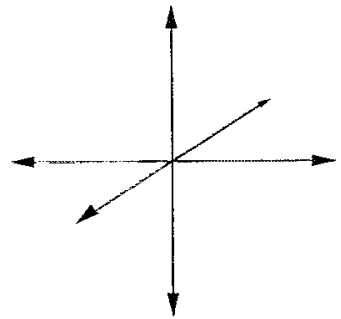

(a)

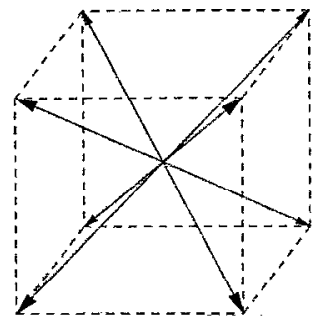

(b)
FIG. 1. Two Broadwell models: (a) single speed particles move only along Cartesian axes, (b) single speed particles move at $45^{\circ}$ to axes.

velocities. Yet, few CA models beside those of Nadiga et $a .^{15}$ and Chen et al. ${ }^{22}$ include multispeed particles.

\section{INTEGERIZED VELOCITIES}

The molecular models treated in the present work were introduced by Goldstein et al. ${ }^{23}$ where the Broadwell model was generalized, and by Goldstein and Sturtevant. ${ }^{24}$ Many details of the model are provided in Goldstein ${ }^{25}$ and large-scale unsteady two-dimensional simulations using the discrete-velocity model are discussed in Goldstein. ${ }^{26}$ In those works the evolution of a flow is modeled, as first described for continuous velocity particles by Bird, ${ }^{27}$ by independently computing molecular motions and collisions in separate phases of each time step. Since those papers described the movement of monatomic hard-sphere discrete-velocity particles and their interaction with boundaries, this paper will concentrate only on the collisions between particles. We note that by employing the model of particle movement, we can treat a variety of schemes that bridge the gaps between the most basic discrete-velocity models, cellular automata, and continuous-velocity approaches. Whereas in Broadwell's original model, only one type of collision and one particle speed are considered, in these recent papers all collisions which exactly conserve mass, momentum, and energy are considered and any discrete particle velocity is possible.

The model is extended here to permit the simulation of mixtures of different species of molecules having different numbers of internal rotational degrees of freedom, different masses and diameters, and that interact through inverse power-law repulsive $\left(1 / r^{\eta}\right)$ force fields. These extensions are based on the incorporation of the Borgnakke-Larsen ${ }^{28}$ phenomenological model of energy exchange and the variable hard-sphere molccular method which Bird ${ }^{29}$ now includes in his model. While the general aim of the present investigations is to use the discrete-velocity models in a Monte Carlo particle simulation of rarefied gas flows, the models may have applications in finite-difference solutions of the discrete Boltzmann equation, ${ }^{12,13}$ Monte Carlo solutions of the collision integral of the Boltzmann equation, ${ }^{30}$ extensions of rarefied flow calculations well into the continuum flow regime, ${ }^{26}$ and simulations of compressible and/or multispecies flow with cellular automata.
The model begins with the following assumptions, the first three of which are also assumed by the direct simulation Monte Carlo (DSMC) approach of Bird. ${ }^{27,31}$

(a) In every particle collision, mass, momentum, and energy are exactly conserved.

(b) Particle collisions are binary (although ternary collisions might later be incorporated to handle chemistry).

(c) Particle scattering is spherically symmetric in the center-of-mass frame as though the molecules were hard spheres. Interparticle force fields are modeled by varying the particle cross sections in the selection of collision pairs. $^{29,32}$

(d) Both before and after each collision, particles may have only discrete-velocity components [i.e., $\mathbf{c}=(i, j, k)$, where $i, j$, and $k$ are integers] and internal energies.

(e) Particles have mass ratios that are rational fractions (i.e., $i / j$ ).

We wish to create a model that can accommodate a variety of realistic gases and produce adequate solutions of rarefied flow problems. It is also desired and expected that the model will produce results that agree with continuous velocity solutions if a fine enough discretization of velocity space is used. The model must accommodate a method of computation that is reasonably efficient in terms of computation time and memory for problems in two or three spatial dimensions. Finally, it is desirable that the model be suited for the new parallel computer architectures.

\section{INITIAL CONDITIONS}

When the velocity components of the molecules are discretized with a unit speed $q$, the velocity distribution function is not continuous but is an approximation consisting of a series of delta functions. For high-temperature gases, the velocity distribution function is "wide," and many molecular speeds occur, while for cold gases, the velocity distribution function is "narrow," so only a few molecular speeds occur. While all integer molecular speeds can be present (up to the value implied by the size of the variable used to store the velocity component on a computer), they occur with a distribution determined by the local temperature.

Consider a collection of $N$ particles having velocities $\left(u_{i}, v_{i}, w_{i}\right)$ where $i$ is the particle index. All particles occupy cells in physical space of volume $V$ small compared to both a flow field scale length and the local mean-free path. Assume for simplicity that there are only two types of particles present: species $a$ particles having a mass of $m_{a}$ and $\zeta_{a}$ internal rotational degrees of freedom, while species $b$ particles are characterized by $m_{b}$ and $\zeta_{b}$. Here $m_{a}, \zeta_{a}, m_{b}$, and $\zeta_{b}$ are all non-negative integers, $m_{a} \leqslant m_{b}$, and $\zeta_{i} \neq 1$. We use the variable hard-sphere gas model of $\mathrm{Bird}^{29,32}$ in which, for the purposes of computing collision probabilities, the particle diameters are changed depending on the relative collision speed to produce the correct temperature dependence of viscosity in an equilibrium continuous velocity gas. In each interspecies collision, however, the ratio of the diameters of a particle of species $a$ to a particle of 
species $b$ is a constant, $D$. The parameter describing the power-law dependence of viscosity on temperature is $\omega$, and it is related to the exponent $\eta$ of an inverse power-law molecular interaction potential by

$$
\omega=2 /(\eta-1) \text {. }
$$

In the Monte Carlo simulations discussed below, the particle cross sections and initial equilibrium gas temperature, $T_{0}$, are normalized with a chosen equilibrium variable hard-sphere mean-free path $\lambda_{0}$ and collision frequency $v_{0}$. From Bird, ${ }^{27,32}$ we find these values to be

$$
\sigma_{a a}=\frac{1}{\lambda_{0} N_{0} \sqrt{2}} P^{\omega} \frac{(2-\omega)^{-\omega}}{\Gamma(2-\omega)}\left(\frac{1}{1+\left(n_{0 b} / n_{0 a}\right) K \sqrt{\frac{1}{2}\left(1+m_{a} / m_{b}\right)}}+\frac{1}{(1 / D)+\left(n_{0 a} / n_{0 b}\right) K \sqrt{\frac{1}{2}\left(1+m_{b} / m_{a}\right)}}\right)=\frac{\sigma_{b b}}{D^{2}},
$$

where $\Gamma$ is the gamma function, $P \equiv T_{0} / T_{\text {ref, }}, T_{\text {ref }}$ is a reference temperature, $K \equiv \frac{1}{4}\left(1+2 / D+1 / D^{2}\right)$, and

$$
\frac{k T_{0}}{q^{2}}=\frac{n_{0}^{2} v_{0}^{2}}{8 \pi} \frac{1}{\left[P^{\omega}(2-\omega)^{\omega} \Gamma(2-\omega)\right]^{2}} \frac{1}{\left\{n_{a}^{2} d_{a a}^{2} \sqrt{\left(2 / m_{a}\right)}+2 n_{a} n_{b} d_{a b}^{2} \sqrt{\left[\left(m_{a}+m_{b}\right) / m_{a} m_{b}\right]}+n_{b}^{2} d_{b b}^{2} \sqrt{2 / m_{b}}\right\}^{2}} .
$$

The particle number density is $n$; subscript zeros refer to the initial equilibrium state; $k$ is the Boltzmann constant and $d$ is a particle diameter $\left(d_{a a}=\sqrt{\sigma_{a a} / \pi}, d_{b b}\right.$ $=\sqrt{\sigma_{b b} / \pi}$.

If $k T / m_{b}=R_{b} T$ is greater than about $2 q^{2}$, it is sufficiently accurate to take the equilibrium distribution of discrete velocities to be equal to the Maxwellian distribution at the same temperature [i.e., $\left.f_{u}(u) \propto \exp \left(-u^{2} m_{b} / 2 k T\right)\right]$. At lower temperatures the discrete-velocity distribution, found by equating the gain and loss collision terms of the discrete Boltzmann equation and iteratively solving the resulting set of algebraic equations, ${ }^{12}$ should be used. The discrete distribution closely resembles a Maxwellian except at the lowest temperatures (e.g., less than $0.5 q^{2}$ ).

\section{CHOICE OF COLLISION PARTNERS}

During a period of time that is small compared to the mean collision time $\left(1 / v_{0}\right)$, the probability that two given particles in volume $V$ will collide is proportional to the fraction of that volume swept out by their trajectories in the center-of-mass frame. Particles on exactly parallel trajectories, possible in a discrete-velocity gas, cannot collide. An acceptance/rejection scheme is used to obtain, on average, the correct equilibrium distribution of collision relative velocities. The collision procedure is to first choose a pair of particles at random. Suppose one particle is of species $a$ and the other of species $b$. The value of $\sigma_{a b} \sqrt{c_{r}^{2}} /\left(\sigma_{a b} \sqrt{c_{r}^{2}}\right)_{\max }$ is then compared to a random number $0<R_{f}<1$ and the particle pair is rejected if $R_{f}$ is greater than this ratio and no collision is counted; a new pair is then chosen. The subscript max indicates the maximum such value found in $V$. Here,

$$
\sigma_{a b}=\pi \frac{1}{4}\left(d_{a a}+d_{b b}\right)^{2}\left\{m_{r} c_{r}^{2} /\left[2(2-\omega) k T_{\text {ref }}\right]\right\}^{-\omega},
$$

a cross section which decreases as the relative collision speed increases; ${ }^{29} m_{r}$ is the reduced mass and

$$
\sqrt{c_{r}^{2}}=\sqrt{\left(u_{a}-u_{b}\right)^{2}+\left(v_{a}-v_{b}\right)^{2}+\left(w_{a}-w_{b}\right)^{2}} .
$$

Since the velocity components are integers, $c_{r}^{2}$ is also an integer and it can be used as an index to access all reasonable values of $\sqrt{c_{r}^{2}}$ and $\sigma_{a b}$ from precomputed arrays.

\section{COLLISION DYNAMICS}

Once the colliding pair has been chosen, the outcome must be determined. In the model gas of polyatomic molecules, a fraction $\chi$ of the collisions will be inelastic-there will be an exchange of internal and translational energies; $\chi$ is the inverse of the rotational collision number. The value of $\chi$ will be taken to be constant ${ }^{29,31}$ although a value that varied with collision energy ${ }^{33}$ and colliding species would be more accurate. For the present, however, we are interested in comparing the discrete-velocity model to the continuous-velocity model and will use the same value of $\chi$ in both. For simplicity, let us first consider the elastic collisions in which no exchange occurs.

\section{A. Elastic collisions}

A collision can be described in a three-dimensional velocity space in which the velocities of the two particles initially lie at two different integer points $\mathbf{u}_{a}$ and $\mathbf{u}_{b}$. In Fig. 2 an example in two dimensions is presented. The relative velocity $\mathbf{c}_{r}$ is $\mathbf{u}_{a}-\mathbf{u}_{b}$ and the center-of-mass velocity $\mathbf{c}_{c m}=\left[1 /\left(m_{a}+m_{b}\right)\right]\left(m_{a} \mathbf{u}_{a}+m_{b} \mathbf{u}_{b}\right)$ lies along the relative velocity, closer to the velocity of the more massive particle. The possible values of $\mathbf{c}_{c m}$ range over $\left[1 /\left(m_{a}+m_{b}\right)\right](i, j, k)$ for all integers $i, j$, and $k$ but do not necessarily take on integer values themselves. They form a subgrid in velocity space on which $\mathbf{c}_{c m}$ may lie which can be used to identify the points on the main grid that are valid post-collision velocities. Because mass and momentum are conserved, $\mathbf{c}_{c m}$ remains unaltered by the collision. Hence, the vector $\mathbf{c}_{r}$ simply pivots around $\mathbf{c}_{c m}$, as shown by the large circles in Fig. 2. For molecules moving in a three-dimensional velocity space, we would be concerned with the analogous spheres indicating the possible collision outcomes.

Since we have supposed that there is no exchange of internal and relative translational energy, $\left|\mathbf{c}_{r}\right|$ also remains unchanged. Because we have assumed [assumption (c), 


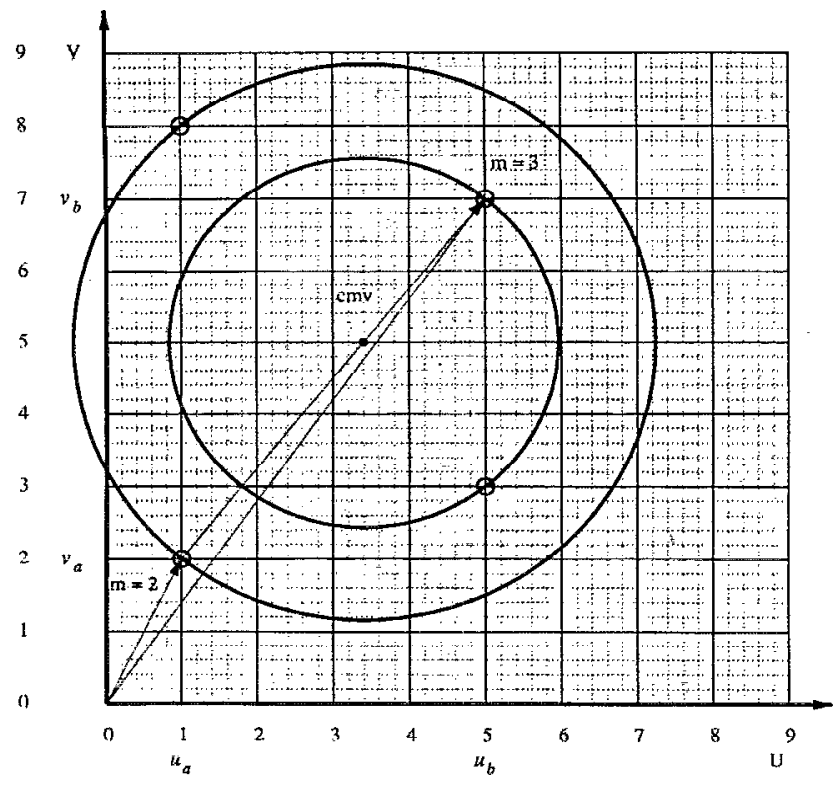

FIG. 2. Valid velocities are indicated by small circles. Solid lines indicate main grid, dotted lines cross at subgrid points. Mass ratio is $2 / 3$, cmv is the center-of-mass velocity.

above] isotropic scattering in the center-of-mass frame, the post-collision particle velocities can simply be chosen randomly from among the discrete points in $(U, V, W)$ which lie on opposing points on the surfaces of the two concentric constant energy circles (spheres in three dimensions) that are centered on $\mathbf{c}_{c m}$. A look-up table containing the points on spheres of a given squared radius that are centered at the origin is precomputed. Here $\left|\mathbf{c}_{r}\right|^{2}$ is used to access the correct size sphere from the table and a random point on the sphere can be rapidly determined. ${ }^{23,25}$ The point is then translated back to its correct location relative to $\mathbf{c}_{\mathrm{cm}}$.

The random choice of points is simply a first approximation to isotropic scattering. It has been found $\mathrm{d}^{29,32,34}$ in continuous-velocity models that whether isotropic scattering or a more accurate determination of the scattering angle is used, there are only small effects on the macroscopic flow quantities. In the discrete-velocity gas, if a sufficient number of discrete points are available on the collision spheres, nearly isotropic scattering occurs for the gas as a whole. ${ }^{23}$ If there are few points, however, the collision outcomes become notably anisotropic and the original velocity, always a possible outcome, is found to be chosen too often. Such a collision is ineffective or null. When a null result is obtained in a multispecies collision we try once more to pick a random point on the sphere. Since each random choice is time consuming and each additional pick provides diminishing returns, we try only this one additional time to pick a non-null point. Sometimes for small collision spheres a null collision is unavoidable.

Let us consider this procedure in more detail with a numerical example. In the sample collision shown in Fig. 2 a mass-2 particle initially has a velocity $\mathbf{u}_{a}=(1 q, 2 q, 0 q)$ while a mass-3 particle has a velocity $\mathbf{u}_{b}=(5 q, 7 q, 0 q)$. The center-of-mass velocity is $\left(3_{5}^{2} q, 5 q, 0 q\right)$. The candidates for outcome discrete velocities are indicated by the small open circles on the perimeter of the large circles. A test postcollision relative velocity $\mathbf{c}_{t}$ is drawn at random from the look-up table for a sphere having a squared radius $=m_{a}^{2} \mathbf{c}_{r}^{2}=164$. That is, we consider a sphere which lies not on the main velocity grid but on the velocity subgrid indicated by the intersections of the fine dotted lines in Fig. 2. The subgrid sphere is centered at $\mathbf{c}_{c m}$ and is the sphere of the heavy particle. [Thus, the coordinates of the heavy particle in the subgrid system $=m_{a} \mathbf{c}_{r}$ $=\left(8 q^{\prime}, 10 q^{\prime}, 0 q^{\prime}\right)$ and the coordinates of the light particle are $=-m_{b} \mathbf{c}_{r}=\left(-12 q^{\prime},-15 q^{\prime}, 0 q^{\prime}\right)$ where a prime indicates subgrid.] If both vectors

$$
\frac{m_{a} \mathbf{c}_{a}+m_{b} \mathbf{c}_{b}-\mathbf{c}_{t}}{\left(m_{a}+m_{b}\right)} \text { and } \frac{m_{b} \mathbf{c}_{t}}{m_{a}}
$$

are composed of only integers, then $c_{t}$ is accepted. Otherwise, a new test point on the same subgrid sphere is chosen. As seen in the figure, in the $(U, V)$ plane the only valid $\mathbf{c}_{r}$ are $(4 q, 5 q, 0 q)$ and $(4 q,-5 q, 0 q)$. The post-collision velocity of the heavy particle is [from Eq. (6)] $\left(m_{a} c_{a}\right.$ $\left.+m_{b} \mathbf{c}_{b}-\mathbf{c}_{t}\right) /\left(m_{a}+m_{b}\right)$; the velocity of the light particle is found from the conservation of momentum. This procedure is time consuming because the acceptable fraction of $\mathbf{c}_{t}$ drops off rapidly as $\left(m_{a}+m_{b}\right) / m$ increases where $m$ is a unit mass. The acceptable fraction also increases slightly with greater velocity resolution for small values of $\left(m_{a}+m_{b}\right) / m$ but decreases slightly for large values of $\left(m_{a}+m_{b}\right) / m$. In this particular two-dimensional example, there are eight intersections of the heavy particle circle with the subgrid $\left[\left( \pm 8 q^{\prime}, \pm 10 q^{\prime}\right)\right.$ and $\left.\left( \pm 10 q^{\prime}, \pm 8 q^{\prime}\right)\right]$, of which only two are acceptable. Much time is saved if the results of all possible division and modulus operations are precomputed and stored in small arrays. While it is possible to reduce the computational time substantially by creating a look-up table containing the results of the entire computational process (the valid values of $\mathbf{c}_{t}$ for all possible $\mathbf{c}_{r}$ and species combinations), the table would probably consume a prohibitive amount of computer memory.

The procedure for this inverse problem of efficiently finding points on the surface of a sphere is simpler if the particles have the same mass. The other points can then be quickly found without resorting to any acceptance/ rejection technique. ${ }^{25}$

\section{B. Collisions with internal energy exchange}

Although the rotational internal energy of the molecules in a real gas is distributed between $\zeta$ degrees of freedom, that distribution does not directly effect particle motions and, hence, does not effect the macroscopic flow quantities. In the present model each particle simply has its three components of velocity and an integer variable indicating the total amount of internal energy. This is termed an energy sink model. ${ }^{31}$

To distribute the collision energy between the internal and translational modes we adopt the method of Bird $^{29}$ who uses the variable hard-sphere molecular model and the Borgnakke-Larsen ${ }^{28}$ phenomenological model of cn- 
ergy exchange. In the Borgnakke-Larsen model the postcollision energy is first distributed between the internal and translational modes with the equilibrium distribution known from theory. The internal energy is then split between the two particles, again based on the equilibrium distribution. The assumption is that while the gas as a whole is not in equilibrium, each collision yields an equilibrium distribution of energies and velocities in the centerof-mass frame.

In the Borgnakke-Larsen model the maximum possible value of $\left|c_{r}^{*}\right|^{2}$ is

$$
\left|\mathbf{c}_{r}^{*}\right|_{\max }^{2}=\left(2 / m_{r}\right)\left(\frac{1}{2} m_{r}\left|\mathbf{c}_{r}\right|^{2}+\epsilon_{a}+\epsilon_{b}\right),
$$

where $\epsilon$ is the internal energy of a molecule. The superscript * indicates a post-collision value. The probability, $P\left(\left|\mathbf{c}_{r}^{*}\right|^{2} /\left|\mathbf{c}_{r}\right|_{\max }^{2}\right)$, of $\left|\mathbf{c}_{r}^{*}\right|^{2} /\left|\mathbf{c}_{r}\right|_{\max }^{2}$ occurring in an equilibrium gas is found in Bird. ${ }^{29}$ To save time, $P\left(\left|\mathbf{c}_{r}^{*}\right|^{2} /\right.$ $\left.\left|c_{r}\right|_{\max }^{2}\right)$ can be precomputed for a modest number of values and stored in an array and the actual value found by interpolation. If a randomly chosen value for $\left|\mathbf{c}_{r}^{*}\right|^{2}$ yields a probability greater than $R_{f}$, that $\left|c_{r}^{*}\right|^{2}$ is accepted. Otherwise a different such $\left|\mathbf{c}_{r}^{*}\right|^{2}$ is selected. On average, this acceptance/rejection procedure yields the equilibrium distribution of energies given by $P\left(\left|\mathbf{c}_{r}^{*}\right|^{2} /\left|\mathbf{c}_{r}\right|_{\max }^{2}\right)$.

Adapting the selection of an appropriate sized collision sphere to the discrete-velocity model is difficult. When the relative translational energy, that is, the size $\left(\left|\mathbf{c}_{r}\right|\right)$ of the spheres is to be changed, the new sphere chosen must have some allowed point(s) on it. For there to be any points on the new sphere, it is necessary and sufficient that the new sphere be of the same class as the original sphere. In using the term "class" we mean that the center-of-mass velocity must lie in the same place in a unit cell in velocity space. That is, if $\left(\left|u_{r}\right|,\left|v_{r}\right|,\left|w_{r}\right|\right) \bmod \left[\left(m_{a}+m_{b}\right) / m\right]=(\alpha, \beta, \gamma)$, then $\left(\left|u_{r}^{*}\right|,\left|v_{r}^{*}\right|,\left|w_{r}^{*}\right|\right) \bmod \left[\left(m_{a}+m_{b}\right) / m\right]$ must also have the same components $\alpha^{*}, \beta^{*}$, and $\gamma^{*}$, though not necessarily in the same order [for example, $\left(\alpha^{*}, \beta^{*}, \gamma^{*}\right)=(\beta, \gamma, \alpha)$ ]. Components $\alpha, \beta$, and $\gamma$ can be integers ranging between 0 and $\left[\left(m_{a}+m_{b}\right) / m\right] / 2$. (If $\alpha \nless\left[\left(m_{a}+m_{b}\right) / m\right] / 2$ then $\alpha \Leftarrow\left[\left(m_{a}+m_{b}\right) / m\right]-\alpha$. Similarly for $\beta$ and $\gamma$. $)$ Values of $\alpha, \beta$, and $\gamma$ all less than 10 are adequate if $\left[\left(m_{a}+m_{b}\right) / m\right]<21$. (As will be seen, larger masses will not be generally practical.) While spheres of a given size may have more than one class, spheres of the same class tend to be spaced evenly in energy (for example, if $\left[\left(m_{a}+m_{b}\right) / m\right]=4$, class $\left(\alpha^{*}, \beta^{*}, \gamma^{*}\right)=(1,2,2)$ spheres may be found at $\left|c_{r}\right|^{2}=9+8 i$ for positive integers $i$ while class $\left(\alpha^{*}, \beta^{*}, \gamma^{*}\right)=(2,2,2)$ spheres are found at $\left.\left|\mathbf{c}_{r}\right|^{2}=12+32 i\right)$.

There may be gaps in this spacing, however, particularly for small spheres [e.g., $\left|\mathbf{c}_{r}\right|^{2}=25$ is not of class $\left.\left(\alpha^{*}, \beta^{*}, \gamma^{*}\right)=(1,2,2)\right]$. Because of this, choosing $\left|\mathbf{c}_{r}^{*}\right|^{2}$ randomly from among those possible produces a bias toward larger values of $\left|\mathbf{c}_{r}^{*}\right|^{2} /\left|\mathbf{c}_{r}\right|_{\text {max }}^{2}$. This bias can be effectively eliminated by weighing the choice of $\left|\mathbf{c}_{r}^{*}\right|^{2}$ by the proximity of the neighboring spheres of the same class. We define this probability as

$$
P_{\text {neigh }}=(A-B) / C \text {, }
$$

where $A=\Delta\left|\mathrm{c}_{r}^{*}\right|^{2}$ to next highest $\left|\mathrm{c}_{r}^{*}\right|^{2}$ with the same class, $B=\Delta\left|\mathbf{c}_{r}^{*}\right|^{2}$ to next lowest $\left|\mathbf{c}_{r}^{*}\right|^{2}$ with the same class, and $C$ is the minimum spacing between $\left|\mathbf{c}_{r}^{*}\right|^{2}$ of this class.

If the particles are of the same mass a second less important type of bias is introduced because $\left|\mathbf{c}_{r}\right|_{\max }^{2}$ is almost always of the same class as $\left|\mathbf{c}_{r}\right|^{2}$ and is thus chosen too frequently; any other value of $\left|\mathbf{c}_{r}^{*}\right|^{2}$ can only be chosen about one time in four. To remedy this bias it is necessary to reject half of the $\left|\mathbf{c}_{r}^{*}\right|^{2}$ choices when $\left|\mathbf{c}_{r}^{*}\right|^{2}=\left|\mathbf{c}_{r}\right|_{\text {max }}^{2}$ This has little effect on the time of computation since it occurs rarely.

Hence, to efficiently find the other spheres from which to choose the post-collision translational energy, $\frac{1}{2} m_{r}\left|\mathbf{c}_{r}^{*}\right|^{2}$, we simply choose randomly from among those spheres of the correct class because the choice is random in the Borgnakke-Larsen model. Each sphere is then subject to acceptance/rejection with the probability $P\left(\left|\mathbf{c}_{r}^{*}\right|^{2} /\right.$ $\left.\left|\mathbf{c}_{r}\right|_{\max }^{2}\right) P_{\text {neigh }}$. The classes of each sphere and the values of $P_{\text {neigh }}$ can be stored in small arrays.

\section{Distributing internal energy between the particles}

The collision energy remaining after the translational energy is accounted for is then split between $\epsilon_{a}$ and $\epsilon_{b}$. Since this energy $\epsilon_{\text {coll }}$ is an integer, $\epsilon_{a}$ and $\epsilon_{b}$ may also be integers. If one particle is monatomic, the other receives all of the internal energy. Otherwise, the probability ${ }^{29}$ of $\epsilon_{a}^{*} / \epsilon_{\text {coll }}$ is used in a simple acceptance/rejection scheme to determine $\epsilon_{a}^{*}$. The complicated probability function $P\left(\epsilon_{a}^{*} / \epsilon_{\text {coll }}\right)$ can again be interpolated from a modest precomputed array. Finally, the remaining internal energy is assigned to $\epsilon_{b}^{*}$.

\section{RESULTS}

We investigate the relaxation of a spatially homogeneous gas to thermal equilibrium and only consider the collision process. The calculations were performed on a SUN Sparcstation. In the first example the gas is a mixture of $\mathrm{CO}_{2}$ and $\mathrm{N}_{2}$. Assume that at $t=0$ the $\mathrm{CO}_{2}$ is ten times hotter than the $\mathrm{N}_{2}$ and that both gases are and will remain rotationally fully excited for the range of temperatures considered. The mean velocity of each gas is zero. As collisions occur, the temperatures of the two gases will equilibrate. The species mass ratio is $7 / 11(\approx 0.64)$, which we will approximate as $2 / 3(\approx 0.67)$. Both species have two rotational degrees of freedom, which is appropriate for a $\mathrm{CO}_{2}$ temperature less than about $120 \mathrm{~K}$. There are equal numbers $(50000)$ of molecules of each species and $\omega=0.33$, $D=0.752 .{ }^{29}$ We assume, as have others, ${ }^{35,36}$ that only one collision in five causes the exchange of internal and translational energy (that is, $\chi=0.2$ ) and that all other collisions are elastic. This probability of exchange is in fair agreement with experimental data. ${ }^{37}$ Four temperatures (for definitions of the different temperatures, see Bird ${ }^{31}$ ), two translational and two rotation, of interest are $T_{N_{2} \text {, trans }}, T_{\mathrm{CO}_{2} \text {,trans }}, T_{\mathrm{N}_{2} \text {,rot }}$, and $T_{\mathrm{CO}_{2} \text {,rot }}$. The evolution of these temperatures, normalized by the equilibrium temperature, with the number of collisions per particle (i.e., one collision time implies $N_{0}$ collisions), is plotted in Fig. 3. 


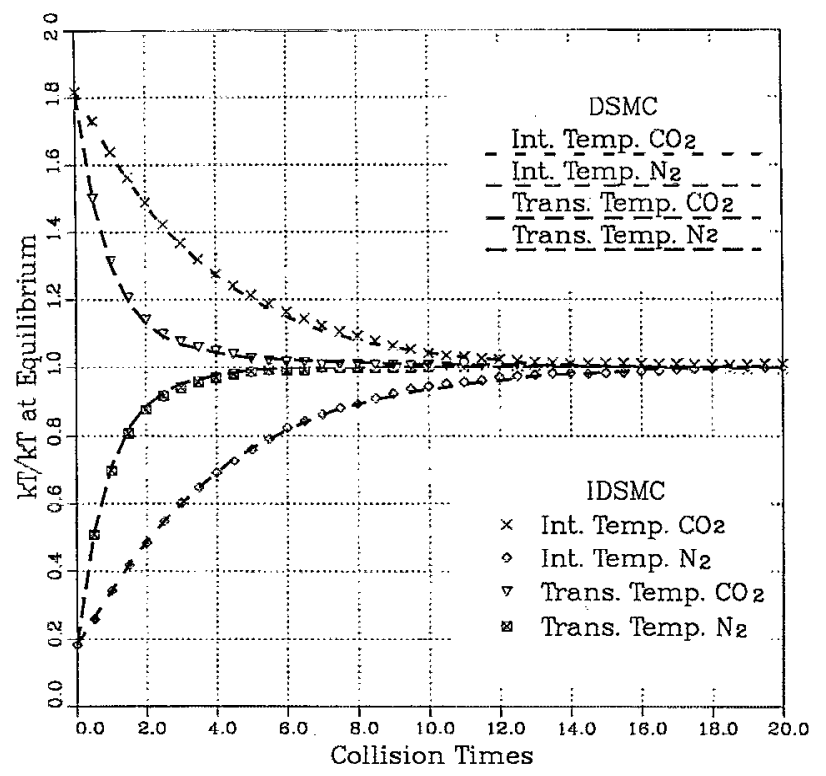

FIG. 3. Relaxation of $\mathrm{CO}_{2}$ and $\mathrm{N}_{2}$ to equilibrium.

Integer velocity (IDSMC) results are compared to the solution found with the continuous-velocity DSMC method of Bird in which the correct particle masses ( 7 and 11) are used. [In a comparison (not shown) between DSMC calculations with a mass ratio of $7 / 11$ and a ratio of $2 / 3$, the solutions agree to within $1 \%$. The solution is found to be fairly insensitive to the exact mass ratio specified.] It is seen that the IDSMC and DSMC solutions correspond very well. Only moderate velocity resolution was necessary ( $k T_{\text {total }}=36.4 q^{2}$ or initially about $\pm 22 q$ velocities in each direction for the $\mathrm{CO}_{2}$ molecules and about $\pm 8 q$ velocities for the $\mathrm{N}_{2}$ molecules) in order to obtain this agreement.

In Fig. 4 is the distribution of discrete internal energies

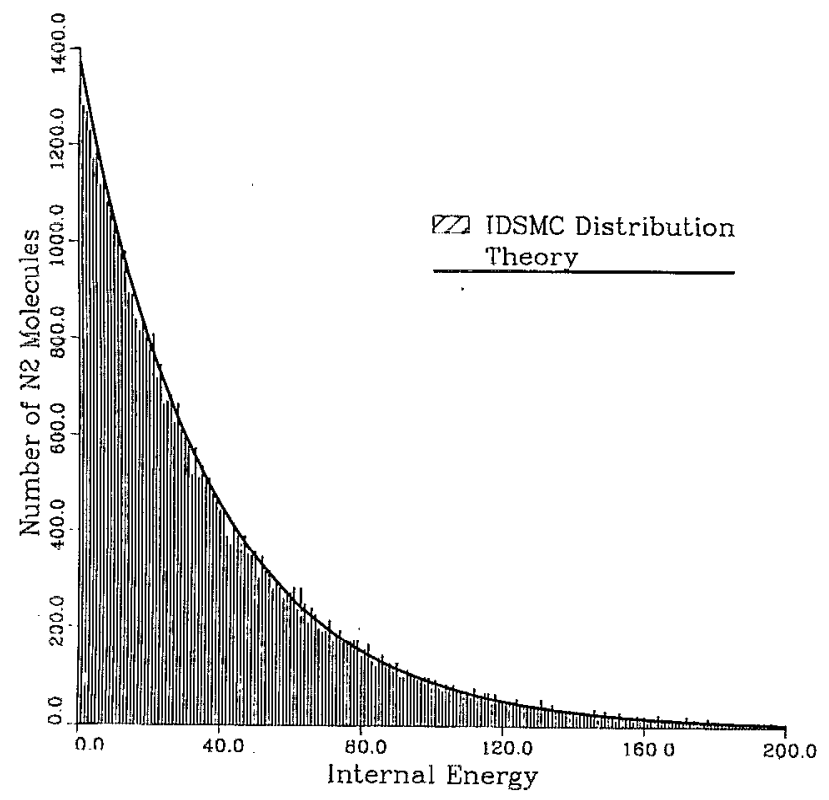

FIG. 4. Equilibrium internal energy distribution of $\mathrm{N}_{2}$.

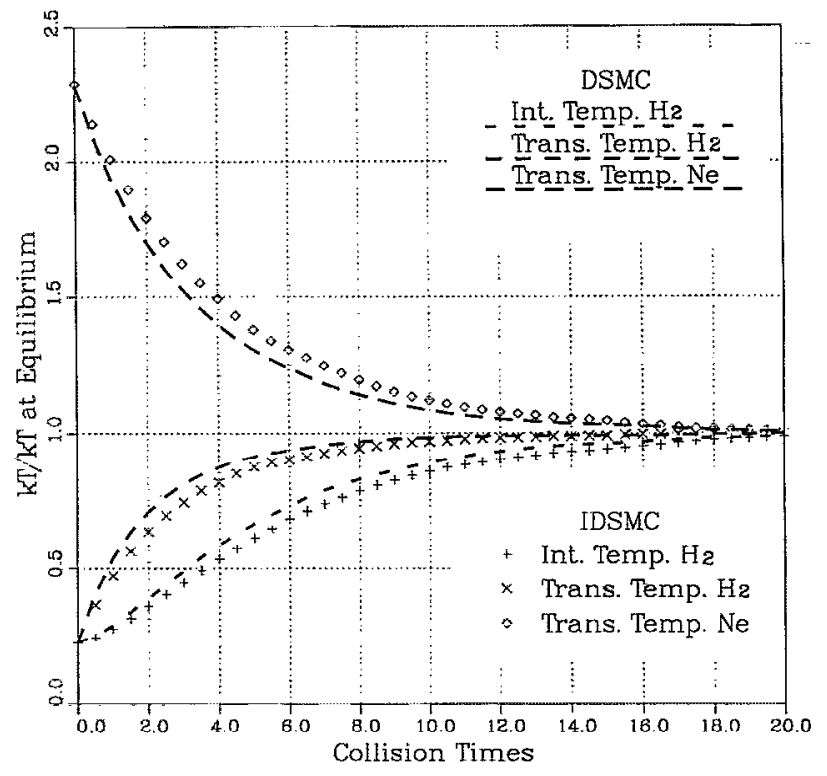

FIG. 5. Relaxation of $\mathrm{H}_{2}$ and $\mathrm{Ne}$ to equilibrium.

for the $\mathrm{N}_{2}$ at equilibrium compared to the theoretical distribution $\left[f(\epsilon) \propto \epsilon^{5 / 2-1} \exp (-\epsilon / k T)\right]$ corresponding to the same total internal energy. Again, the results agree well.

Consider now the simulation of the relaxation to equilibrium of a mixture of $\mathrm{H}_{2}$ and $\mathrm{Ne}$ in which the ratio of particle masses is $1: 10$. The hydrogen has two rotational degrees of freedom and the neon has none. Again we simulate 100000 molecules, $\omega=0.16$ and $D=1.06$. Even though high velocity resolution was used $\left(k T_{\text {total }}=206 q^{2}\right.$ or initially $\pm 32 q$ velocities in each direction for both the $\mathrm{H}_{2}$ and the Ne molecules), in Fig. 5 it is clear that the IDSMC molecules relax too slowly. This error is due to the large mass ratio that produces multispecies collisions in which the inner collision sphere for the more massive $\mathrm{Ne}$ has few intersections with the discrete velocity lattice and many of the collisions are null. Often, in fact, only the original velocity is a possible outcome. Increasing the velocity resolution reduces the error. As in the first example, the probability of exchange of internal and translational energy $\chi$ is again chosen to be 0.2 .

When many of the collisions are null, the assumption that the relative velocity is rotated through a random angle is invalid and the collision results are anisotropic. To a certain extent, however, the occurrence of a zero deflection collision represents simply a small deflection collision so some null collisions are allowable. Figure 6 shows how the fraction of null collisions in the mixture as a whole varies with the velocity resolution for the three different gas mixtures (at equilibrium) discussed herein. In low-speed collisions there are simply few points on the collision spheres and a null collision is likely. The probability of a null collision decreases with increasing velocity resolution because the average size of the collision spheres increases (i.e., the mean relative collision energy $\propto T$ ). Other measures of collision anisotropy are given in Refs. 23 and 25. In a 


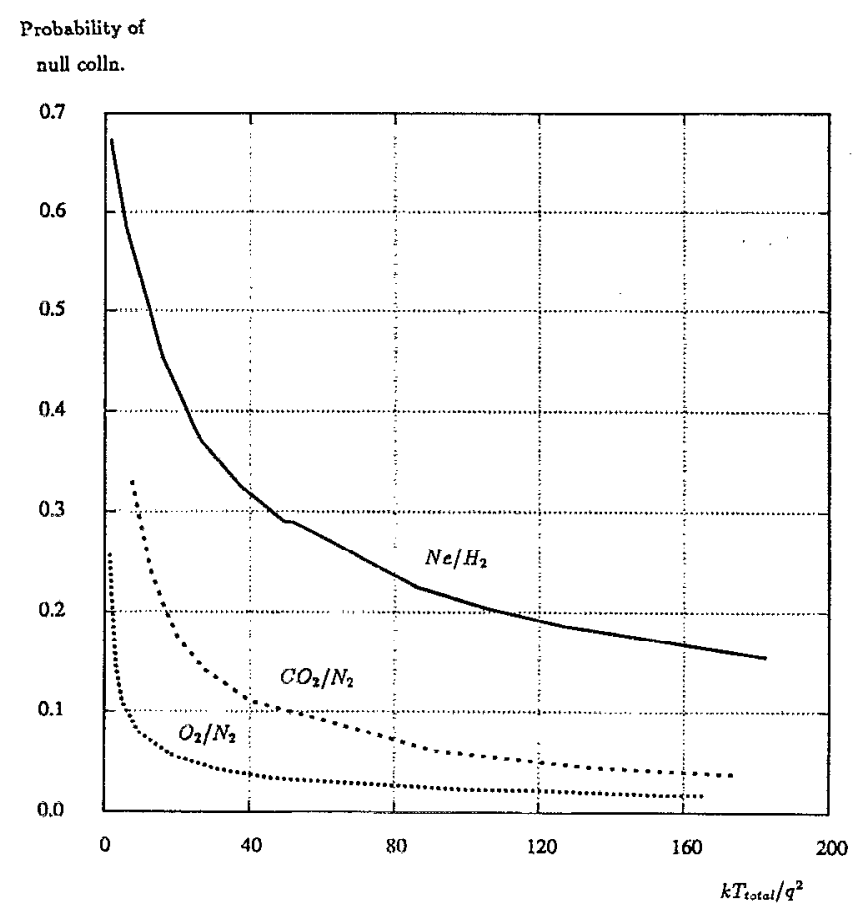

FIG. 6. Probability of a collision being null in the three gas mixtures.

collision in which there is an internal energy exchange, the outcome relative speed is nearly always different from the input so virtually none of those collisions are null. Thus if the probability of exchange were greater, the IDSMC and DSMC results would correspond better. Indeed, for $\chi=1$ even with a mass ratio of ten, the discrete- and continuousvelocity results are nearly indistinguishable (probability of null collisions is about 0.016 ). A value of $\chi$ that high is unrealistic, however.

\section{DISCUSSION}

A discrete-velocity model of particle collisions in rarefied flows has been studied theoretically and through numerical experiments. The investigation was motivated by a desire to understand the fundamental physics of a discretevelocity gas and by the need to link discrete-velocity molecular models to accepted continuous velocity models. A new molecular model has been developed to permit such a linkage and has been implemented in the integer direct simulation Monte Carlo method. The method, in combination with earlier particle movement models, may also be an acceptable engineering tool to extend particle simulations toward the continuum flow regime.

The above procedures use simple integer arithmetic throughout the whole collision operation, whereas in the standard DSMC approach a substantial number of floating point operations are needed. We note that interpolated values from look-up tables and lists of random numbers may be used in the DSMC method as well. The basic DSMC method, however, runs more slowly than the IDSMC on computers which are not highly optimized for floating point operations. Table I illustrates the speed up ( $\equiv$ IDSMC collision rate/DSMC collision rate) of the col-
TABLE I. Speed-up obtained with the IDSMC method versus the DSMC method.

\begin{tabular}{lccc}
\hline \hline Computer & $\mathrm{O}_{2} / \mathrm{N}_{2}$ & $\mathrm{CO}_{2} / \mathrm{N}_{2}$ & $\mathrm{H}_{2} / \mathrm{Ne}$ \\
\hline SUN 3/50 & 5.1 & 4.0 & 3.3 \\
SUN Sparcstation & 2.5 & 1.8 & 1.7 \\
SGI 4D/240 & 1.6 & 1.2 & 0.9 \\
Cray YMP & 1.2 & 0.9 & 0.6 \\
\hline \hline
\end{tabular}

lision process obtained with the integer approach for different gas combinations on four different computers. The programs were written in $\mathrm{C}$ and were compiled with the maximum compiler optimization available. On multiprocessor machines only a single processor was used.

The differences between computers indicate the improved relative floating point performance of the more powerful machines. Also evident is that for the higher mass ratio gas combinations the IDSMC required many rejections of trial relative velocities and thus ran slower. As discussed below, IDSMC simulations of oxygen and nitrogen assumed the molecular masses were equal. Both codes, but the IDSMC in particular, would benefit substantially by having faster integer random number generators. On a computer with enough memory, tables of pseudorandom numbers might be used instead of subroutine calls.

With floating point numbers, the storage per particle for a full three(spatial)-dimensional calculation is 31 bytes (12 for the 3 components of velocity, 12 for 3 positions, 4 for internal energy, 2 for the particle index, and 1 for the particle type), while with the discrete velocity approach it is $14(3,6,2,2$, and 1$)$. Thus, for memory-limited computations with the IDSMC, flows with up to 2.2 times larger Reynolds numbers can be calculated.

How accurately the discrete velocity model can represent rarefied gas flows depends upon how many points there are on the collision spheres. Fewer than ten velocity components (i.e., \pm 5 in each direction $U, V, W$ ) have been found adequate for simulations with a single species monatomic gas. ${ }^{23}$ Increased values of $\left[\left(m_{a}+m_{b} / m\right)\right]$ produce a marked decrease in the number of collision possibilities available. Figure 7 shows the average number of points on all collision spheres up to $\left|\mathbf{c}_{r}\right|^{2}=100$ as a function of $\left[\left(m_{a}+m_{b}\right) / m\right] /$ gcd where gcd is the greatest common denominator of $m_{a}$ and $m_{b}$. More often than not, for values of $\left[\left(m_{a}+m_{b}\right) / m\right]>7$ on these small spheres there is only one point and the collision must be null. In a simulation in which more than $10 \%$ to $20 \%$ of the collisions are null, there is an increase in the diffusivity in the gas which in turn affects the macroscopic flow properties (e.g., shock waves thickness). Because the size of the look-up table grows as $\left(\left|\mathbf{c}_{r}\right|_{\max } m_{a}\right)^{3}$, the high velocity resolution that would be required for large values of $\left[\left(m_{a}+m_{b}\right) / m\right]$ is not presently attainable. For example, with the largest look-up table used here the entire code took about 3 words on the Cray, about one-eighth of its memory. Hence, while some real gas combinations can now be simulated [i.e., $\mathrm{H}_{2} / \mathrm{D}_{2}$ $\left(m_{a} / m_{b}=2 / 4=1 / 2\right), \quad \mathrm{H}_{2} / \mathrm{DH}(=2 / 3), \quad \mathrm{CO}_{2} / \mathrm{N}_{2}(=11 / 7$ $\left.\approx 3 / 2), \mathrm{O}_{2} / \mathrm{He}(=4 / 1), \mathrm{N}_{2} / \mathrm{H}_{2}(=7 / 1)\right]$ other combina- 


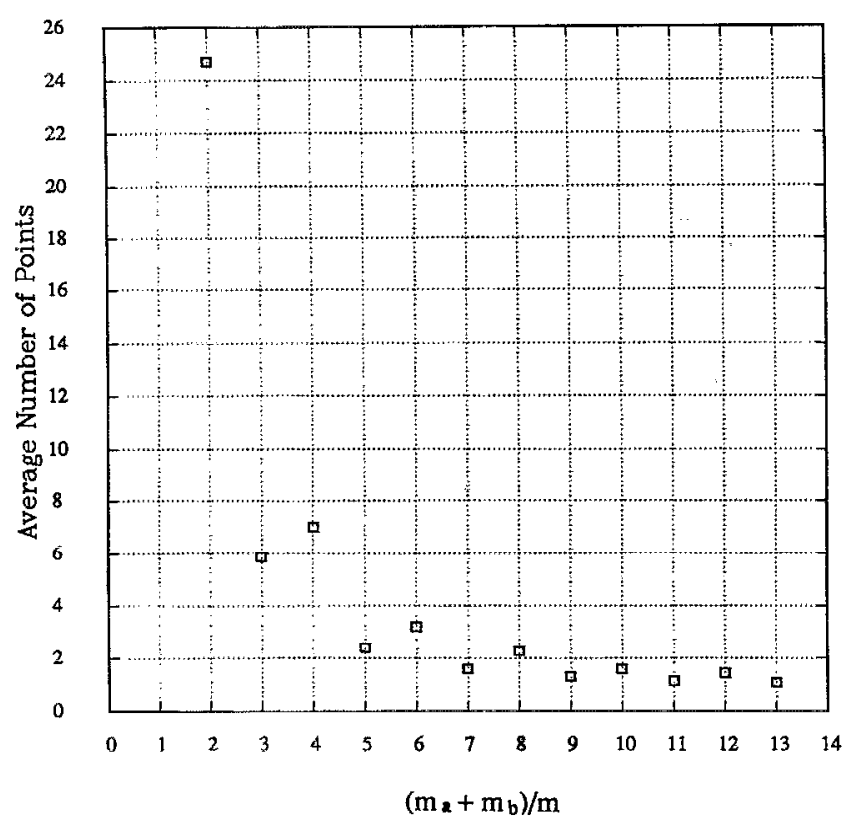

FIG. 7. Average number of intersections of all spheres with squared radii less than $100 q^{2}$.

tions in which the mass ratio cannot be approximated by a ratio of small integers will remain out of reach as long as a look-up table is needed. In particular, a mixture of $\mathrm{O}_{2} / \mathrm{N}_{2}(=8 / 7)$, which is of practical significance, is just beyond the capability of present course-grained parallel hypercube computers when the look-up table is repeated in every processor. On the Cray a mixture of $\mathrm{O}_{2} / \mathrm{N}_{2}$ can be simulated but the most powerful new computers have parallel architectures and are the machines best suited for this kind of simulation of fluid flow. Approximating the $\mathrm{O}_{2}$ and $\mathrm{N}_{2}$ masses as equal, however, is adequate for some purposes. For example, in a simulation of thermal relaxation similar to those shown above, the error obtained by using equal mass particles in the IDSMC simulation rather than a mass ratio of $8 / 7$, used in the DSMC, is very small (Fig. 8 ) and may be smaller than the error introduced with the uncertainty in the other physical parameters, i.e., $\omega$ and $\chi$. (In fact, the choice of a constant value for $\chi$ rather than one which depends on $\left|\mathbf{c}_{r}\right|^{2}$ probably produces a greater error. ${ }^{33}$ ) Without a low mass ratio approximation, computations on a parallel processor would require a look-up table distributed between the processors. What is needed is to minimize storage requirements by eliminating or reducing the size of the look-up table by finding another way to quickly answer the question: "Given a particular collision sphere radius and center-of-mass location in velocity space, what are the intersections of the sphere with an integer lattice?" or even, "What is one randomly chosen intersection of such a sphere with the lattice?"

The current model assumes that the number of rotational degrees of freedom of a molecule is constant. While the energy levels in the present model are discrete, the phenomenological model assumes a form of averaging and is only applicable to fully excited modes having many

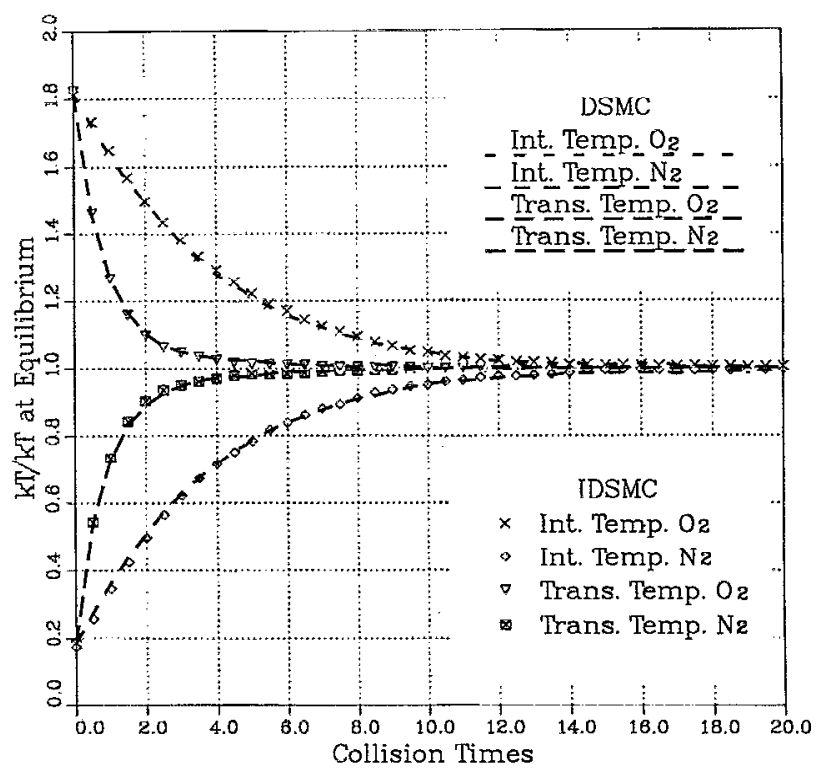

FIG. 8. Relaxation of $\mathrm{O}_{2}$ and $\mathrm{N}_{2}$ to equilibrium.

quantum states. Thus, the model may be applied to polyatomic gases over only a modest range of temperatures in which the ratio of specific heats, $\gamma$, is nearly constant. For example, $\gamma=1.4$ for $\mathrm{N}_{2}$ only for $T<500 \mathrm{~K}$. At the lowest temperatures, rotational energy should be considered from the quantum-mechanical point of view. At higher temperatures internal vibration should be considered.

The method of incorporating rotational degrees of freedom can be generalized to include vibrational and electronic modes as well. ${ }^{38,39}$ The simulation of vibrational degrees of freedom is a prerequisite for any accurate simulation of chemistry, including such simple chemistry as dissociation/recombination. The method of changing the collision sphere sizes and using an acceptance/rejection procedure could remain unchanged. It appears, however, that because the energy changes due to vibrational excitation are so large compared to those of rotation, there could again be constraints on the possible species combinations because of the requirement for a reasonably sized look-up table. Nonetheless, for a modest range of temperatures the present discrete-velocity approach without vibrational degrees of freedom can provide quantitatively accurate results.

\section{ACKNOWLEDGMENTS}

Suggestions and support from Professor Bradford Sturtevant at Caltech are greatly appreciated. Part of this work was completed at the Center for Non-Linear Studies at Los Alamos National Lab, documented in Report No. LA-UR 90-3243. Part of the computation was done at the Pittsburgh Supercomputer Center.

The work presented here was also in part supported by the Office of Naval Research under Award No. N0001491-J-1588. 
${ }^{1} S$. Chandrasekhar, Radiative Transfer (Dover, New York, 1980).

'J. J. Duderstadt and W. R. Martin, Transport Theory (Wiley, New York, 1979).

${ }^{3}$ E. P. Gross, "Recent investigations of the Boltzmann equation," Rarefied Gas Dynamics: Proceedings of the First International Symposium, Nice (Pergamon, New York, 1960).

${ }^{4} \mathrm{~J}$. E. Broadwell, "Study of rarefied shear flows by the discrete velocity method," Space Technology Laboratories, Inc., Report No. 9813-6001RUO00, 1963.

${ }^{5}$ J. E. Broadwell, "Study of rarefied shear flow by the discrete velocity method," J. Fluid Mech. 19, 401 (1964).

'J. E. Broadwell, "Shock structure in a simple discrete velocity gas," Phys. Fluids 7, 1243 (1964).

${ }^{7}$ R. E. Caflisch and G. Papanicolaou, "The fluid dynamic limit of a nonlinear Boltzmann equation," Comments Pure Appl. Math. 32, 589 (1979).

${ }^{8}$ R. E. Caflisch, "Navier-Stokes and Boltzmann shock profiles for a model of gas dynamics," Comments Pure Appl. Math. 32, 521 (1979).

${ }^{9} \mathrm{R}$. Gatignol, "Théorie cinétique des gaz à répartition discrète de vitesses," in Lecture Notes in Physics (Springer-Verlag, Berlin, 1975), Vol. 36.

${ }^{10} \mathrm{~T}$. Platkowski and R. Illner, "Discrete velocity models of the Boltzmann equation: A survey of the mathematical aspects of theory," SIAM Rev. 30, 213 (1988).

"G. R. McNamera and G. Zanetti, "Using the Boltzmann equation to simulate lattice gas automata," Phys. Rev. Lett. 61, 2332 (1988).

${ }^{12} \mathrm{~T}$. Inamuro and B. Sturtevant, "Heat transfer in a discrete-velocity gas," in Rarefied Gas Dynamics, Procecdings of 17th Intcrnational Symposium on Rarefied Gas Dynamics, Aachen, edited by A. E. Beylich (VCH Verlagsgesellschaft, Weinheim, 1990).

${ }^{13}$ T. Inamuro and B. Sturtevant, "Numerical study of discrete-velocity gases, “ Phys. Fluids A 2, 2196 (1990).

${ }^{14}$ L. P. Kadanoff, G. McNamera, and G. Zanetti, "From automata to fluid flow: Comparisons of simulation and theory," Phys. Rev. A 40, 4527 (1989).

${ }^{15}$ B. T. Nadiga, J. E. Broadwell, and B. Sturtevant, "Study of a multispeed cellular automaton," in Rarefied Gas Dynamics: Theoretical and Computational Techniques, Proceedings of 16th International Symposium on Rarefied Gas Dynamics, Pasadena, CA, edited by E. P. Muntz, D. P. Weaver, and D. H. Campbell (AIAA, Washington, DC, 1989); Prog. Aeronaut. Astronaut. 118, 155 (1989).

${ }^{16}$ L. N. Long, R. M. Coopersmith, and B. G. McLachlan, "Cellular automatons applied to gas dynamic problems," presented at the AIAA 19th Fluid Dynamics, Plasma Dynamics and Lasers Conference, Honolulu, Hawaii, 8-10 June 1987.

${ }^{17}$ G. Doolen (Editor), Lattice Gas Methods for Partial Differential Equations (Addison-Wesley, Reading, MA, 1990).

${ }^{18}$ D. Dab, A. Lawniczak, J. Boon, and R. Kapral, "Cellular-automaton model for reactive systems," Phys. Rev. Lett. 64, 2462 (1990).

${ }^{19} \mathrm{D}$. H. Rothman, "Macroscopic laws for immiscible two-phase flow in porous media: Results from numerical experiments," J. Geophys. Res. B 95, 8663 (1990).
${ }^{20} \mathrm{G}$. Zanetti, "The hydrodynamics of lattice gas automata," Phys. Rev. A 40, 1539 (1989).

${ }^{21}$ U. Frisch, B. Hasslacher, and Y. Pomeau, "A lattice gas automaton for the Navier-Stokes equation," Phys. Rev. Lett. 56, 1505 (1986).

${ }^{22} \mathrm{~S}$. Chen, H. Chen, and G. D. Doolen, "How the lattice gas model for the Navier-Stokes equation improves when a new speed is added," Complex Syst. 3, 243 (1989).

${ }^{23}$ D. Goldstein, B. Sturtevant, and J. E. Broadwell, "Investigations of the motion of discretc-velocity gascs," in Ref. 15; Prog. Acronaut. Astronaut. 118, 100 (1989).

${ }^{24}$ D. Goldstein and B. Sturtevant, "Discrete velocity gasdynamics simulations in a parallel processing environment," presented at the Thermophysics Conference, Buffalo, NY, 12-14 June 1989; AIAA Paper No. AIAA-89-1668, 1989.

${ }^{25} \mathrm{D}$. Goldstein, "Investigations of a discrete velocity gas," $\mathrm{Ph} . \mathrm{D}$. thesis, California Institute of Technology, 1990.

${ }^{26} \mathrm{D}$. Goldstein, "Near continuum applications of a discrete-velocity gas model," in Ref. 12.

${ }^{27} \mathrm{G}$. A. Bird, "The structure of normal shock waves in a binary gas mixture," J. Fluid Mech. 31, 657 (1968).

${ }^{28}$ C. Borgnakke and P. S. Larsen, "Statistical collision model for Monte Carlo simulation of polyatomic gas mixtures," J. Comput. Phys. 18, 405 (1975).

${ }^{29} \mathrm{G}$. A. Bird, "Monte-Carlo simulation in an engineering context," Progress in Aeronautics and Astronautics, Twelfth International Symposium on Rarefied Gasdynamics, 7-11 July 1980 (AIAA, New York, 1981), pp. 239-255.

${ }^{30}$ A. Nordsieck and B. Hicks, "Monte Carlo evaluation of the Boltzmann collision integral," in Rarefied Gas Dynamics, Proceedings of 5th Symposium on Rarefied Gas Dynamics, Oxford, England, edited by C. L. Brundin (Academic, New York, 1967), pp. 695-710.

${ }^{31}$ G. A. Bird, Molecular Gas Dynamics (Clarendon, Oxford, 1976).

${ }^{32}$ G. A. Bird, "Definition of mean free path for real gases," Phys. Fluids 26, 3222 (1983)

${ }^{33}$ I. D. Boyd, "Rotational-translational energy transfer in rarefied nonequilibrium flows," Phys. Fluids A 2, 447 (1990).

${ }^{34}$ D. Erwin, G. Phan-Van-Diep, and E. P. Muntz, "Nonequilibrium gas flows. I: A detailed validation of Monte Carlo direct simulation for monatomic gases," Phys. Fluids A 3, 697 (1991).

${ }^{35} \mathrm{~F}$. Bergemann, "Monte Carlo simulation of the hypersonic mon- and diatomic gas flow past a disk at an angle of attack," in Ref. 12.

${ }^{36} \mathrm{~T}$. Shimada, "Direct simulation of hypersonic rarefied flows about atmospheric reentry capsules," in Ref. 12.

${ }^{37}$ A. E. Belikov, G. I. Sukhinin, and R. G. Sharafutdinov, "Rate constants for $\mathrm{R}-\mathrm{T}$ relaxation of $\mathrm{N}_{2}$ in argon supersonic jets," in Ref. 15: Prog. Aeronaut. Astronaut. 117, 52 (1989).

${ }^{38}$ G. A. Bird, "Direct sîmulation of typical AOTV entry flows," presented at the AIAA.ASME 4th Joint Thermophysics and Heat Transfer Conference, Boston; AIAA Paper No. AIAA-86-1310, 1986

${ }^{39} \mathrm{G}$. A. Bird, "Simulation of multi-dimensional and chemically reacting flows," in Rarefied Gas Dynamics, Proccedings of the 11th International Symposium on Rarefied Gas Dynamics, edited by R. Campargue, (CEA, Paris, 1979), Vol. 1. 